\title{
组合扰动界: II. 极分解
}

\section{黎稳 $^{1 *}$ 孙伟伟 $^{2}$}

(1. 华南师范大学数学科学学院, 广州 $510631 ; 2$. 香港城市大学数学系, 香港)

摘要 本文旨在研究极分解 $A=Q H$ 的扰动界, 其中 $Q$ 是酉矩阵和 $H$ 是 Hermite 半正定 矩阵. 此前人们已经分别得到了酉极因子, Hermite 极因子和 $A$ 的奇异值的最优 (渐近) 扰动 界为:

$$
\sigma_{r}^{2}\|\Delta Q\|_{F}^{2} \leqslant\|\Delta A\|_{F}^{2}, \frac{1}{2}\|\Delta H\|_{F}^{2} \leqslant\|\Delta A\|_{F}^{2} \text { 和 }\|\Delta \Sigma\|_{F}^{2} \leqslant\|\Delta A\|_{F}^{2},
$$

其中 $\Sigma=\operatorname{diag}\left(\sigma_{1}, \sigma_{2}, \ldots, \sigma_{r}, 0, \ldots, 0\right)$ 并且 $\sigma_{r}$ 表示矩阵 $A$ 最小的非零奇异值. 本文我们给出 如下组合的扰动界

$$
\sigma_{r}^{2}\|\Delta Q\|_{F}^{2}+\frac{1}{2}\|\Delta H\|_{F}^{2} \leqslant\|\Delta A\|_{F}^{2}
$$

和

$$
\sigma_{r}^{2}\|\Delta Q\|_{F}^{2}+\|\Delta \Sigma\|_{F}^{2} \leqslant\|\Delta A\|_{F}^{2} .
$$

上述两个渐近界对其中的每个因子来说都是最优的. 另外, 也给出相应的绝对扰动界.

关键词 极分解 扰动 奇异值

$\operatorname{MSC}(2000)$ 主题分类 $65 \mathrm{~F} 10,15 \mathrm{~A} 18,05 \mathrm{C} 87$

\section{1 引言}

用 $\mathcal{C}^{m \times n}$ 表示 $m \times n$ 复矩阵集合并用 $\mathcal{C}_{r}^{m \times n}$ 表示秩为 $r$ 的 $m \times n$ 复矩阵集合. 本文总是 假定 $m \geqslant n$. 用 $\|\cdot\|_{2},\|\cdot\|_{F}$ 和 $\|\cdot\|$ 分别表示矩阵的谱范数、Frobenius 范数和酉不变范数. 设 $A$ 的奇异值分解可表示为

$$
A=U\left(\begin{array}{cc}
\Sigma_{1} & 0 \\
0 & 0
\end{array}\right) V^{*}
$$

并记

$$
H=V_{1} \Sigma_{1} V_{1}^{*}, \quad Q=U_{1} V_{1}^{*},
$$

收稿日期: 2005-06-02; 接受日期: 2007-04-12

国家自然科学基金 (批准号: 10671077), 广东省自然科学基金 (批准号: 06025061, 031496) 和香港特别行政区 研究基金 (批准号: CityU 102204) 资助项目

*E-mail: liwen@scnu.edu.cn; maweiw@mathh.cityu.edu.hk 
其中 $U=\left(U_{1}, U_{2}\right) \in \mathcal{C}^{m \times m}$ 和 $V=\left(V_{1}, V_{2}\right) \in \mathcal{C}^{n \times n}$ 是酉阵, $U_{1} \in \mathcal{C}_{r}^{m \times r}, V_{1} \in \mathcal{C}_{r}^{n \times r}, \Sigma_{1}=$ $\operatorname{diag}\left(\sigma_{1}, \ldots, \sigma_{r}\right), \sigma_{i}(i=1,2, \ldots, r)$ 是 $A$ 的奇异值且满足 $\sigma_{1} \geqslant \sigma_{2} \geqslant \cdots \geqslant \sigma_{r}>0$ 和 $*$ 表示矩 阵的共轭转置, 则矩阵 $A$ 的极分解为

$$
A=Q H .
$$

$Q$ 和 $H$ 分别称为 $A$ 的酉极因子和 Hermite 半正定极因子, 有时我们使用 $|A|$ 表示矩阵 $A$ 的 Hermite 半正定极因子. 对 $r<n$, 则满足条件

$$
R\left(Q^{*}\right)=R(H)
$$

的极分解 (1.1) 是唯一的 (见文献 [1]). 当 $r=m=n$ 时, 有关极分解的应用可参见文献 [2]. 本文总假定极分解都满足唯一性条件.

许多学者已经研究了极因子的扰动界 ${ }^{[1,3-13]}$. 设 $\widetilde{A}$ 是 $A$ 的扰动矩阵且 $\widetilde{A}=\widetilde{Q} \widetilde{H}$ 是 $\widetilde{A}$ 的极分解. 对两个极因子的扰动界有如下 (渐近) 结论:

$$
\frac{1}{2}\|H-\widetilde{H}\|_{F}^{2} \leqslant\|A-\widetilde{A}\|_{F}^{2}
$$

和

$$
\sigma_{r}^{2}\|Q-\widetilde{Q}\|_{F}^{2} \leqslant\|A-\widetilde{A}\|_{F}^{2} .
$$

一般地, 这两个界是最优的. 然而, 由于上述界是在只考虑一个极因子的扰动情形下得到的, 因 此很容易找到例子说明这两个界中的一个不是精确的. 本文的目的是给出酉极因子、Hermite 半正定极因子或奇异值的组合扰动界, 得到如下两个新的结果:

$$
\sigma_{r}^{2}\|Q-\widetilde{Q}\|_{F}^{2}+\frac{1}{2}\|H-\widetilde{H}\|_{F}^{2} \leqslant\|A-\widetilde{A}\|_{F}^{2}
$$

和

$$
\sigma_{r}^{2}\|Q-\widetilde{Q}\|_{F}^{2}+\|\Sigma-\widetilde{\Sigma}\|_{F}^{2} \leqslant\|A-\widetilde{A}\|_{F}^{2} .
$$

注意到 Mirsky 定理 (例如, 见文献 [14, 15])

$$
\|\Sigma-\widetilde{\Sigma}\|_{F}^{2} \leqslant\|A-\widetilde{A}\|_{F}^{2},
$$

于是组合界 (1.4) 和 (1.5) 式对每个极因子都是最优的.

\section{2 预备}

设 $A, \widetilde{A} \in \mathcal{C}_{r}^{m \times n}(m \geqslant n)$ 的奇异值分解分别为

$$
A=U \Sigma V^{*}, \quad \widetilde{A}=\widetilde{U} \widetilde{\Sigma} \widetilde{V}^{*},
$$

其中

$$
\Sigma=\left(\begin{array}{cc}
\Sigma_{1} & 0 \\
0 & 0
\end{array}\right) \in \mathcal{C}_{r}^{m \times n}, \quad \widetilde{\Sigma}=\left(\begin{array}{cc}
\widetilde{\Sigma}_{1} & 0 \\
0 & 0
\end{array}\right) \in \mathcal{C}_{r}^{m \times n}
$$

$\Sigma_{1}=\operatorname{diag}\left(\sigma_{1}, \ldots, \sigma_{r}\right), \widetilde{\Sigma}_{1}=\operatorname{diag}\left(\widetilde{\sigma}_{1}, \ldots, \widetilde{\sigma}_{r}\right), \sigma_{1} \geqslant \cdots \geqslant \sigma_{r}>0$ 及 $\widetilde{\sigma}_{1} \geqslant \cdots \geqslant \widetilde{\sigma}_{r}>0$.

设 $I_{p}$ 是 $p \times p$ 单位矩阵并记

$$
I_{m, n}^{(p)} \equiv\left(\begin{array}{cc}
I_{p} & 0 \\
0 & 0
\end{array}\right)
$$


为简单起见用 $I^{(p)}$ 表示 $I_{m, n}^{(p)}$. 将 $S=\widetilde{U}^{*} U$ 和 $T=\widetilde{V}^{*} V$ 写成如下的分块形式:

$$
S=\left(\begin{array}{cc}
S_{11} & S_{12} \\
S_{21} & S_{22}
\end{array}\right) \in \mathcal{C}^{m \times m}, \quad T=\left(\begin{array}{cc}
T_{11} & T_{12} \\
T_{21} & T_{22}
\end{array}\right) \in \mathcal{C}^{n \times n},
$$

其中 $S_{11}$ 和 $T_{11}$ 是 $r \times r$ 矩阵, 则 $S$ 和 $T$ 是酉矩阵. 记

$$
M=2 I-S_{11}^{*} T_{11}-T_{11}^{*} S_{11}, \quad \widetilde{M}=2 I-T_{11} S_{11}^{*}-S_{11} T_{11}^{*} .
$$

用 $m_{i j}$ 和 $\widetilde{m}_{i j}$ 分别表示矩阵 $M$ 和 $\widetilde{M}$ 的第 $(i, j)$ 元. 则 $M$ 和 $\widetilde{M}$ 是 Hermite 半正定矩阵.

文献 $[10,11]$ 证明了以下基本性质:

$$
\begin{aligned}
& \|Q-\widetilde{Q}\|_{F}^{2}=\left\|S I^{(r)}-I^{(r)} T\right\|_{F}^{2}=\operatorname{tr}(M)=\operatorname{tr}(\widetilde{M}), \\
& \|A-\widetilde{A}\|=\|S \Sigma-\widetilde{\Sigma} T\|,
\end{aligned}
$$

其中 $\operatorname{tr}(A)$ 表示矩阵 $A$ 的迹. 记

$$
\Gamma=\Sigma-\sigma I^{(r)}, \quad \widetilde{\Gamma}=\widetilde{\Sigma}-\sigma I^{(r)},
$$

则有

$$
\|S \Sigma-\widetilde{\Sigma} T\|_{F}^{2}=\sigma^{2}\|Q-\widetilde{Q}\|_{F}^{2}+\|S \Gamma-\widetilde{\Gamma} T\|_{F}^{2}+2 \sigma \mathcal{R} e \operatorname{tr}\left[\left(S I^{(r)}-I^{(r)} T\right)(S \Gamma-\widetilde{\Gamma} T)^{*}\right],
$$

其中 $\operatorname{Re}$ 表示一个复数的实部.

$\mathrm{Li}^{[8]}$ 证明了: 当 $\sigma \leqslant \min \left\{\sigma_{r}, \widetilde{\sigma}_{r}\right\}$ 时, $\operatorname{Re} \operatorname{tr}\left[\left(S I^{(r)}-I^{(r)} T\right)(S \Gamma-\widetilde{\Gamma} T)^{*}\right]$ 是非负的. 并由此 导出如下的扰动界:

$$
\|Q-\widetilde{Q}\|_{F} \leqslant \frac{1}{\min \left\{\sigma_{r}, \widetilde{\sigma}_{r}\right\}}\|A-\widetilde{A}\|_{F} .
$$

最近我们对 (2.3) 式作了更进一步的研究, 证明了 ${ }^{[10]}$ : 对 $A, \widetilde{A} \in \mathcal{C}_{r}^{m \times n}$ 有

$$
\begin{aligned}
\|A-\widetilde{A}\|_{F}^{2} \geqslant & \sigma^{2}\|Q-\widetilde{Q}\|_{F}^{2}+\|S \Gamma-\widetilde{\Gamma} T\|_{F}^{2}+\sigma\left(\sigma_{r-1}+\widetilde{\sigma}_{r-1}-2 \sigma\right) \operatorname{tr}(M) \\
& -\sigma\left(\sigma_{r-1}-\sigma_{r}\right) m_{r r}-\sigma\left(\widetilde{\sigma}_{r-1}-\widetilde{\sigma}_{r}\right) \widetilde{m}_{r r} .
\end{aligned}
$$

引理 2.1 [4, 定理 VII. 5.7] 设 $B$ 和 $\widetilde{B}$ 是任意两个矩阵. 则

$$
\||B|-|\widetilde{B}|\|_{F}^{2}+\left\|\left|B^{*}\right|-\left|\widetilde{B}^{*}\right|\right\|_{F}^{2} \leqslant 2\|B-\widetilde{B}\|_{F}^{2},
$$

其中 $|*|$ 表示矩阵 $*$ 的 Hermite 半正定极因子.

显然, $|A|=H$ 和 $|\widetilde{A}|=\widetilde{H}$. 记 $H^{\prime}=U \Sigma^{*} U^{*}$ 和 $\widetilde{H}^{\prime}=\widetilde{U} \widetilde{\Sigma}^{*} \widetilde{U}^{*}$, 则 $H^{\prime}$ 和 $\widetilde{H}^{\prime}$ 分别是 $A^{*}$ 和 $\widetilde{A}^{*}$ 的 Hermite 半正定极因子, 即, $\left|A^{*}\right|=H^{\prime}$ 和 $\left|\widetilde{A}^{*}\right|=\widetilde{H}^{\prime}$.

引理 2.2 如果 $\sigma=\min \left\{\sigma_{r}, \widetilde{\sigma}_{r}\right\}$, 那么有

$$
\|S \Gamma-\widetilde{\Gamma} T\|_{F}^{2} \geqslant \frac{1}{2}\|H-\widetilde{H}\|_{F}^{2}+\frac{1}{2}\left\|H^{\prime}-\widetilde{H}^{\prime}\right\|_{F}^{2}
$$

和

$$
\|S \Gamma-\widetilde{\Gamma} T\|_{F}^{2} \geqslant \frac{1}{2}\|H-\widetilde{H}\|_{F}^{2}+\frac{1}{2}\|\Sigma-\widetilde{\Sigma}\|_{F}^{2} .
$$

证明 不失一般性, 假定 $\sigma_{r} \leqslant \widetilde{\sigma}_{r}$, 则 $\sigma=\sigma_{r}$. 易知

$$
\|S \Gamma-\widetilde{\Gamma} T\|_{F}=\left\|U \Gamma V^{*}-\widetilde{U} \widetilde{\Gamma} \widetilde{V}^{*}\right\|_{F}=\|B-\widetilde{B}\|_{F},
$$

其中 $B=U \Gamma V^{*}$ 和 $\widetilde{B}=\widetilde{U} \widetilde{\Gamma} \widetilde{V}^{*}$. 由 $\sigma-\sigma_{r} \leqslant 0$ 和 $\sigma-\widetilde{\sigma}_{r} \leqslant 0$ 知 $B=U \Gamma V^{*}$ 和 $\widetilde{B}=\widetilde{U} \widetilde{\Gamma} \widetilde{V}^{*}$ 分 别为 $B$ 和 $\widetilde{B}$ 的奇异值分解, 并且有

$$
\||B|-|\widetilde{B}|\|_{F}=\left\|V \Gamma V^{*}-\widetilde{V} \widetilde{\Gamma} \widetilde{V}^{*}\right\|_{F}
$$




$$
\begin{aligned}
& =\left\|\widetilde{V}^{*} V \Gamma-\widetilde{\Gamma} \widetilde{V}^{*} V\right\|_{F} \\
& =\left\|\widetilde{V}^{*} V \Sigma-\widetilde{\Sigma} \widetilde{V}^{*} V\right\|_{F} \\
& =\|H-\widetilde{H}\|_{F} .
\end{aligned}
$$

类似地,

$$
\left\|\left|B^{*}\right|-\left|\widetilde{B}^{*}\right|\right\|_{F}=\left\|H^{\prime}-\widetilde{H}^{\prime}\right\|_{F} .
$$

因而由引理 2.1 可得 $(2.6)$ 式.

因为 $\left|B^{*}\right|=U \Gamma^{*} U^{*}$ 和 $\left|\widetilde{B}^{*}\right|=\widetilde{U} \widetilde{\Gamma}^{*} \widetilde{U}^{*}$, 由 Mirsky 定理 (1.6), 我们有

$$
\left\|\left|B^{*}\right|-\left|\widetilde{B}^{*}\right|\right\|_{F}^{2}=\left\|U \Gamma^{*} U^{*}-\widetilde{U} \widetilde{\Gamma}^{*} \widetilde{U}^{*}\right\|_{F}^{2} \geqslant\|\Gamma-\widetilde{\Gamma}\|_{F}^{2}=\|\Sigma-\widetilde{\Sigma}\|_{F}^{2} .
$$

由引理 2.1 和 (2.8) 式可得 (2.7) 式.

\section{3 组合扰动界}

在 (2.4) 式中取 $\sigma=\sigma_{r}=\min \left\{\sigma_{r}, \widetilde{\sigma}_{r}\right\}$, 可得

$$
\|A-\widetilde{A}\|_{F}^{2} \geqslant \sigma_{r} \widetilde{\sigma}_{r}\|Q-\widetilde{Q}\|_{F}^{2}+\|S \Gamma-\widetilde{\Gamma} T\|_{F}^{2},
$$

在此, 我们已经使用了 $(2.2)$ 式和

$$
m_{r r} \leqslant\|Q-\widetilde{Q}\|_{F}^{2}, \quad \widetilde{m}_{r r} \leqslant\|Q-\widetilde{Q}\|_{F}^{2} .
$$

由引理 2.2 我们获得如下定理:

定理 3.1 设 $A, \widetilde{A} \in \mathcal{C}_{r}^{m \times n}$ 的奇异值分解如 (2.1) 式所示. 则

$$
\sigma_{r} \widetilde{\sigma}_{r}\|Q-\widetilde{Q}\|_{F}^{2}+\frac{1}{2}\|H-\widetilde{H}\|_{F}^{2}+\frac{1}{2}\left\|H^{\prime}-\widetilde{H}^{\prime}\right\|_{F}^{2} \leqslant\|A-\widetilde{A}\|_{F}^{2} .
$$

由 Mirsky 定理 (1.6) 有

$$
\|\Sigma-\widetilde{\Sigma}\|_{F}^{2} \leqslant\|H-\widetilde{H}\|_{F}^{2}, \quad\|\Sigma-\widetilde{\Sigma}\|_{F}^{2} \leqslant\left\|H^{\prime}-\widetilde{H}^{\prime}\right\|_{F}^{2},
$$

和

$$
\|\Sigma-\widetilde{\Sigma}\|_{F}^{2} \leqslant \frac{1}{2}\|H-\widetilde{H}\|_{F}^{2}+\frac{1}{2}\left\|H^{\prime}-\widetilde{H}^{\prime}\right\|_{F}^{2},
$$

从而得到如下推论:

推论 3.1 设 $A, \widetilde{A} \in \mathcal{C}_{r}^{m \times n}$ 的奇异值分解如 (2.1) 式所示. 则

$$
\sigma_{r} \widetilde{\sigma}_{r}\|Q-\widetilde{Q}\|_{F}^{2}+\|\Sigma-\widetilde{\Sigma}\|_{F}^{2} \leqslant\|A-\widetilde{A}\|_{F}^{2} .
$$

推论 3.2 在推论 3.1 的条件下, 渐近扰动界 (1.4) 和 (1.5) 成立.

显然组合界 (3.1) 和 (3.2) 比以往的界更加精确. 随后的例子说明 (3.1) 和 (3.2) 式的等 号可以成立.

例 3.1 设 $A$ 是 $2 \times 2$ 矩阵并且 $A=U \Sigma V^{*}$ 是它的奇异值分解, 其中

$$
\Sigma=\left(\begin{array}{cc}
\sigma_{1} & 0 \\
0 & \sigma_{2}
\end{array}\right), \quad \sigma_{1}>\sigma_{2} .
$$

设 $\widetilde{A}=U \widetilde{\Sigma}(V(I+D))^{*}$, 其中

$$
\widetilde{\Sigma}=\left(\begin{array}{cc}
\sigma_{1} & 0 \\
0 & \widetilde{\sigma}_{2}
\end{array}\right), \quad \sigma_{1} \geqslant \widetilde{\sigma}_{2}>0, \quad \widetilde{\sigma}_{2} \neq \sigma_{2},
$$


和

$$
D=\left(\begin{array}{cc}
0 & 0 \\
0 & -a+i \sqrt{2 a-a^{2}}
\end{array}\right), \quad 0<a<2 .
$$

则 $\widetilde{U}=U, \widetilde{V}=V(I+D)$. 经简单的计算可得

$$
\begin{aligned}
& \|Q-\widetilde{Q}\|_{F}=\left\|U D^{*} V^{*}\right\|_{F}=\left\|D^{*}\right\|_{F}=\sqrt{2 a}, \\
& \|H-\widetilde{H}\|_{F}=\left\|H^{\prime}-\widetilde{H}^{\prime}\right\|_{F}=\left|\widetilde{\sigma}_{2}-\sigma_{2}\right|, \\
& \|A-\widetilde{A}\|_{F}=\left\|\Sigma-\widetilde{\Sigma}(I+D)^{*}\right\|_{F}=\sqrt{\left(\widetilde{\sigma}_{2}-\sigma_{2}\right)^{2}+2 \widetilde{\sigma}_{2} \sigma_{2} a} .
\end{aligned}
$$

因而 (3.1) 和 (3.2) 式的等号成立.

注 3.1 因为渐近扰动界 (1.3) 和 (1.4) 对每个因子都是最优的, 所以它们的界也是最优 的. 相应的绝对扰动界显得要复杂些. 目前对西极因子最好的绝对扰动界是

$$
\left(\frac{\sigma_{r}+\widetilde{\sigma}_{r}}{2}\right)^{2}\|Q-\widetilde{Q}\|_{F}^{2} \leqslant\|A-\widetilde{A}\|_{F}^{2},
$$

文献 $[9,10]$ 分别对 $r=m=n$ 和 $r \leqslant \min \{m, n\}$ 情形证明了 (3.3) 式成立. 文献 [9-11] 说明对 任意的扰动来说界 (3.3) 是最优的. 上面的例子也说明了组合界

$$
\left(\frac{\sigma_{r}+\widetilde{\sigma}_{r}}{2}\right)^{2}\|Q-\widetilde{Q}\|_{F}^{2}+\frac{1}{2}\|H-\widetilde{H}\|_{F}^{2} \leqslant\|A-\widetilde{A}\|_{F}^{2}
$$

不成立. 为了得到更好的绝对组合界, 需要对扰动作出限制.

定理 3.2 设 $A, \widetilde{A} \in \mathcal{C}_{r}^{m \times n}$ 有奇异值分解 (2.1). 如果

$$
\|A-\widetilde{A}\|_{F} \leqslant \frac{\sigma_{r}+\widetilde{\sigma}_{r}}{2\left|\sigma_{r}-\widetilde{\sigma}_{r}\right|}\|\Sigma-\widetilde{\Sigma}\|_{F}
$$

那么

$$
\frac{1}{2}\left(\sigma_{r}^{2}+\widetilde{\sigma}_{r}^{2}\right)\|Q-\widetilde{Q}\|_{F}^{2}+\frac{1}{2}\|H-\widetilde{H}\|_{F}^{2} \leqslant\|A-\widetilde{A}\|_{F}^{2} .
$$

证明 不失一般性, 设 $\sigma_{r} \leqslant \widetilde{\sigma}_{r}$. 由 (3.3) 式可知条件 (3.4) 蕴含着

$$
\|Q-\widetilde{Q}\|_{F} \leqslant \frac{1}{\left|\sigma_{r}-\widetilde{\sigma}_{r}\right|}\|\Sigma-\widetilde{\Sigma}\|_{F},
$$

因而在 (2.4) 工中取 $\sigma=\sigma_{r}$ 和由引理 2.2 可得

$$
\begin{aligned}
\|A-\widetilde{A}\|_{F}^{2} \geqslant & {\left[\sigma_{r}^{2}+\sigma_{r}\left(\sigma_{r-1}+\widetilde{\sigma}_{r-1}-2 \sigma_{r}\right)-\sigma_{r}\left(\sigma_{r-1}-\sigma_{r}\right)-\sigma_{r}\left(\widetilde{\sigma}_{r-1}-\widetilde{\sigma}_{r}\right)\right]\|Q-\widetilde{Q}\|_{F}^{2} } \\
& +\frac{1}{2}\|H-\widetilde{H}\|_{F}^{2}+\frac{1}{2}\|\Sigma-\widetilde{\Sigma}\|_{F}^{2} \\
\geqslant & \sigma_{r} \widetilde{\sigma}_{r}\|Q-\widetilde{Q}\|_{F}^{2}+\frac{1}{2}\left(\sigma_{r}-\widetilde{\sigma}_{r}\right)^{2}\|Q-\widetilde{Q}\|_{F}^{2}+\frac{1}{2}\|H-\widetilde{H}\|_{F}^{2} \\
\geqslant & \frac{1}{2}\left(\sigma_{r}^{2}+\widetilde{\sigma}_{r}^{2}\right)\|Q-\widetilde{Q}\|_{F}^{2}+\frac{1}{2}\|H-\widetilde{H}\|_{F}^{2} .
\end{aligned}
$$

从而定理结论成立.

在扰动很小的假设下, 文献 [11] 对 (3.3) 式做了更进一步的改进, 得到如下扰动界:

$$
\frac{1}{2}\left(\sigma_{r}^{2}+\widetilde{\sigma}_{r}^{2}\right)\|Q-\widetilde{Q}\|_{F}^{2} \leqslant\|A-\widetilde{A}\|_{F}^{2} .
$$

显然, (3.5) 式是最优界 (1.2) 和上面扰动界的组合.

接下来考虑当 $A, \widetilde{A} \in \mathcal{C}_{n}^{n \times n}$ 时两个极因子的组合扰动. 这时 (3.4) 式可以进一步减弱. 不 失一般性, 以下总假定 $\sigma_{n} \leqslant \widetilde{\sigma}_{n}$. 
定理 3.3 设 $A, \widetilde{A} \in \mathcal{C}_{n}^{n \times n}$ 有奇异值分解 (2.1). 如果

$$
\|A-\widetilde{A}\|_{2} \leqslant \frac{\sigma_{n}+\widetilde{\sigma}_{n}}{2},
$$

则

$$
\min \left\{\sigma_{n}\left(\sigma_{n-1}+\widetilde{\sigma}_{n-1}-\sigma_{n}\right), \frac{1}{2}\left(\sigma_{n}^{2}+\widetilde{\sigma}_{n}^{2}\right)\right\}\|Q-\widetilde{Q}\|_{F}^{2}+\frac{1}{2}\|H-\widetilde{H}\|_{F}^{2} \leqslant\|A-\widetilde{A}\|_{F}^{2} .
$$

证明 在 (2.4) 式中令 $\sigma=\sigma_{n}$. 由引理 2.2 可得

$$
\begin{aligned}
\|A-\widetilde{A}\|_{F}^{2} \geqslant & \sigma_{n}^{2}\|Q-\widetilde{Q}\|_{F}^{2}+\frac{1}{2}\|H-\widetilde{H}\|_{F}^{2}+\frac{1}{2}\|\Sigma-\widetilde{\Sigma}\|_{F}^{2}+\sigma_{n}\left(\sigma_{n-1}+\widetilde{\sigma}_{n-1}-2 \sigma_{n}\right) \operatorname{tr}(M) \\
& -\sigma_{n}\left(\sigma_{n-1}-\sigma_{n}\right) m_{n n}-\sigma_{n}\left(\widetilde{\sigma}_{n-1}-\widetilde{\sigma}_{n}\right) \widetilde{m}_{n n} .
\end{aligned}
$$

注意到 $[10]$

$$
m_{n n} \leqslant\|Q-\widetilde{Q}\|_{2}^{2}, \quad \widetilde{m}_{n n} \leqslant\|Q-\widetilde{Q}\|_{2}^{2},
$$

从而由 (2.2) 式可得

$$
\begin{aligned}
\|A-\widetilde{A}\|_{F}^{2} \geqslant & \sigma_{n}\left(\sigma_{n-1}+\widetilde{\sigma}_{n-1}-\sigma_{n}\right)\|Q-\widetilde{Q}\|_{F}^{2}+\frac{1}{2}\left(\sigma_{n}-\widetilde{\sigma}_{n}\right)^{2} \\
& \quad-\sigma_{n}\left(\sigma_{n-1}-\sigma_{n}+\widetilde{\sigma}_{n-1}-\widetilde{\sigma}_{n}\right)\|Q-\widetilde{Q}\|_{2}^{2}+\frac{1}{2}\|H-\widetilde{H}\|_{F}^{2} .
\end{aligned}
$$

由文献 [9] 和 (3.6) 式可得

$$
\|Q-\widetilde{Q}\|_{2} \leqslant \frac{2}{\sigma_{n}+\widetilde{\sigma}_{n}}\|A-\widetilde{A}\|_{2} \leqslant 1
$$

于是有

$$
\begin{aligned}
\|A-\widetilde{A}\|_{F}^{2} \geqslant & \sigma_{n}\left(\sigma_{n-1}+\widetilde{\sigma}_{n-1}-\sigma_{n}\right)\|Q-\widetilde{Q}\|_{F}^{2} \\
& -\left[\sigma_{n}\left(\sigma_{n-1}+\widetilde{\sigma}_{n-1}\right)-\frac{3}{2} \sigma_{n}^{2}-\frac{1}{2} \widetilde{\sigma}_{n}^{2}\right]\|Q-\widetilde{Q}\|_{2}^{2}+\frac{1}{2}\|H-\widetilde{H}\|_{F}^{2} .
\end{aligned}
$$

如果

$$
\sigma_{n}\left(\sigma_{n-1}+\widetilde{\sigma}_{n-1}\right)-\frac{3}{2} \sigma_{n}^{2}-\frac{1}{2} \widetilde{\sigma}_{n}^{2} \geqslant 0
$$

则 (3.8) 式可以写成

$$
\|A-\widetilde{A}\|_{F}^{2} \geqslant \frac{1}{2}\left(\sigma_{n}^{2}+\widetilde{\sigma}_{n}^{2}\right)\|Q-\widetilde{Q}\|_{F}^{2}+\frac{1}{2}\|H-\widetilde{H}\|_{F}^{2} .
$$

如果

$$
\sigma_{n}\left(\sigma_{n-1}+\widetilde{\sigma}_{n-1}\right)-\frac{3}{2} \sigma_{n}^{2}-\frac{1}{2} \widetilde{\sigma}_{n}^{2}<0
$$

则

$$
\|A-\widetilde{A}\|_{F}^{2} \geqslant \sigma_{n}\left(\sigma_{n-1}+\widetilde{\sigma}_{n-1}-\sigma_{n}\right)\|Q-\widetilde{Q}\|_{F}^{2}+\frac{1}{2}\|H-\widetilde{H}\|_{F}^{2} .
$$

由上面的最后两个不等式可知 (3.7) 式成立.

注 3.2 条件 (3.4) 和 (3.6) 不能去掉. 例如, $A$ 和 $\widetilde{A}$ 是例 3.1 所定义的矩阵, 且满足 $a>\frac{1}{2}$ 和 $\sigma_{1} \sigma_{2}>\tilde{\sigma}_{2}^{2}$, 则

$$
\|A-\widetilde{A}\|_{F}=\|A-\widetilde{A}\|_{2}>\sqrt{\left(\widetilde{\sigma}_{2}-\sigma_{2}\right)^{2}+\widetilde{\sigma}_{2} \sigma_{2}} \geqslant\left(\widetilde{\sigma}_{2}+\sigma_{2}\right) / 2
$$

由此可得 $\sigma_{2}\left(\sigma_{1}+\tilde{\sigma}_{1}-\sigma_{2}\right)>\frac{1}{2}\left(\sigma_{2}^{2}+\tilde{\sigma}_{2}^{2}\right)$ 和

$$
\frac{1}{2}\left(\sigma_{2}^{2}+\widetilde{\sigma}_{2}^{2}\right)\|Q-\widetilde{Q}\|_{F}^{2}+\frac{1}{2}\|H-\widetilde{H}\|_{F}^{2}>\|A-\widetilde{A}\|_{F}^{2} .
$$


推论 3.3 在定理 3.3 的条件下, 如果

$$
\widetilde{\sigma}_{n}^{2} \leqslant \sigma_{n} \widetilde{\sigma}_{n-1},
$$

则组合扰动界 $(3.5)$ 成立.

\section{4 结束语}

关于矩阵 $A$ 的极分解 $A=Q H$, 我们给出了酉极因子、Hermite 半正定极因子和奇异值 的组合扰动界. 由于所得到的组合界对每个因子都具有最优特性, 因此组合界也有最优特性. 文献 [16] 表明对矩阵的谱分解和奇异值分解也有类似的组合扰动界. 然而, 并非对矩阵的所 有分解都有这样的组合界. 下面的例子说明对矩阵的 $Q R$ 分解不具备此性质. 当 $A, \widetilde{A} \in \mathcal{C}_{n}^{n \times n}$ 时, 由文献 [7] 知道 $Q$ 和 $R$ 因子的扰动界为

$$
\frac{\sigma_{n}^{2}}{2}\|\widetilde{Q}-Q\|_{F}^{2} \leqslant\|\widetilde{A}-A\|_{F}^{2}
$$

和

$$
\frac{1}{\kappa_{R}^{2}(A)}\|\widetilde{R}-R\|_{F}^{2} \leqslant\|\widetilde{A}-A\|_{F}^{2}
$$

例 4.1 设 $Q=I, R=I, A=Q R=I$ 和 $\widetilde{A}=\widetilde{Q} \widetilde{R}$, 其中

$$
\widetilde{Q}=\left(\begin{array}{cc}
1-\delta & -\sqrt{2 \delta-\delta^{2}} \\
\sqrt{2 \delta-\delta^{2}} & 1-\delta
\end{array}\right), \quad \widetilde{R}=\left(\begin{array}{cc}
1 & \sqrt{2 \delta-\delta^{2}} \\
0 & 1
\end{array}\right) .
$$

则

$$
\begin{aligned}
& \|\widetilde{A}-A\|_{F}^{2}=\left\|\left(\begin{array}{cc}
-\delta & -\delta \sqrt{2 \delta-\delta^{2}} \\
\sqrt{2 \delta-\delta^{2}} & \delta-\delta^{2}
\end{array}\right)\right\|_{F}^{2}=2 \delta+\delta^{2} \\
& \|\widetilde{Q}-Q\|_{F}^{2}=4 \delta \\
& \|\widetilde{R}-R\|_{F}^{2}=2 \delta-\delta^{2} \\
& \kappa_{R}(A)=1 .
\end{aligned}
$$

由此可知仅当 $c(A) \equiv 0$ 时, 组合界

$$
\frac{\sigma_{2}^{2}}{2}\|\widetilde{Q}-Q\|_{F}^{2}+c(A)\|\widetilde{R}-R\|_{F}^{2} \leqslant\|\widetilde{A}-A\|_{F}^{2}
$$

才成立.

致谢 作者感谢审稿人的评论和修改建议.

\section{参考文 献}

1 Sun J G, Chen C H. Generalized polar decomposition. Math Numer Sinica, 11: 262-273 (1989)

2 Higham N J. Computing the polar decomposition-with applications. SIAM J Sci Statist Comput, 7: 11601174 (1986)

3 Barrlund A. Perturbation bounds on the polar decomposition. BIT, 30: 101-113 (1989)

4 Bhatia R. Matrix Analysis. New York: Springer, 1997

5 Bhatia R, Mukherjea K. Variation of the unitary part of a matrix. SIAM J Matrix Anal Appl, 15: 10071014 (1994) 
6 Chatelin F, Gratton S. One the condition numbers associated with the polar factorization of a matrix. Numer Linear Algebra Appl, 7: 337-354 (2000)

7 Chang X C, Paige C C, Stewart G W. Perturbation analysis for the $Q R$ decomposition. SIAM J Matrix Anal Appl, 18: 775-791 (1997)

8 Li R C. A perturbation bounds for the generalized polar decomposition. BIT, 33: 304-308 (1993)

9 Li R C. New Perturbation bounds for the unitary polar factor. SIAM J Matrix Anal Appl, 16: 327-332 (1995)

$10 \mathrm{Li}$ W, Sun W W. Perturbation bounds for unitary and subunitary polar factors. SIAM J Matrix Anal Appl, 23: 1183-1193 (2002)

11 Li W, Sun W W. New perturbation bounds for unitary polar factors. SIAM J Matrix Anal Appl, 25: 362-372 (2003)

12 Chen X S, Li W, Sun W W. Some new perturbation bounds for the generalized polar decomposition. BIT, 44: 237-244 (2004)

13 Mathias R. Perturbation bounds for the polar decomposition. SIAM J Matrix Anal Appl, 14: 588-593 (1993)

14 Horn R A, Johnson C R. Matrix Analysis. Cambridge: Cambridge University Press, 1985

15 Stewart G W, Sun J G. Matrix Perturbation Theory. Boston: Academic Press, 1990

16 Li W, Sun W W. Combined perturbation bounds: I. Eigensystems and singular value decomposition. SIAM J Matrix Anal Appl, In press 